\title{
Genetic Contribution of Catechol- $O$-Methyltransferase Polymorphism (Val158Met) in Children With Chronic Tension-Type Headache
}

\author{
CÉSAR FERNÁNDEZ-DE-LAS-PEÑAS, SILVIA AMBITE-QUESADA, INÉS RIVAS-MARTÍNEZ, RICARDO ORTEGA-SANTIAGO, \\ ANA ISABEL DE-LA-LLAVE-RINCÓN, DANIEL M. FERNÁNDEZ-MAYORALAS, AND JUAN A. PAREJA

\begin{abstract}
Department of Physical Therapy, Occupational Therapy, Rehabilitation and Physical Medicine [C.F.-P., R.O.-S., A.I.L.-R.], Department of Anatomy [S.A.-Q., I.R.-M.], Universidad Rey Juan Carlos, Alcorcón, Madrid 28922, Spain; Department of Pediatrics, [D.M.F.-M.], Hospital Quirón, Pozuelo, Madrid 28223, Spain; Department of Neurology [J.A.P.], Fundación Hospital Alcorcón,
\end{abstract}

Alcorcón, Madrid 28922, Spain

\begin{abstract}
Our aim was to investigate the relationship between Val158Met polymorphisms, headache, and pressure hypersensitivity in children with chronic tension-type headache (CTTH). A casecontrol study with blinded assessor was conducted. Seventy children with CTTH associated with pericranial tenderness and 70 healthy children participated. After amplifying Val158Met polymorphism by polymerase chain reactions, we assessed genotype frequencies and allele distributions. We classified children according to their Val158Met polymorphism: Val/Val, Val/Met, Met/Met. Pressure pain thresholds (PPT) were bilaterally assessed over the temporalis, upper trapezius, second metacarpal, and tibialis anterior muscles. The distribution of Val158Met genotypes was not significantly different ( $p=0.335$ ), between children with CTTH and healthy children, and between boys and girls $(p=0.872)$. Children with CTTH with the Met/Met genotype showed a longer headache history compared with those with $\mathrm{Met} / \mathrm{Val}(p=0.001)$ or $\mathrm{Val} / \mathrm{Val}(p=0.002)$ genotype. Children with CTTH with Met/Met genotype showed lower PPT over upper trapezius and temporalis muscles than children with CTTH with Met/Val or Val/Val genotype $(p<0.01)$. The Val158Met catechol-Omethyltransferase (COMT) polymorphism does not appear to be involved in predisposition to suffer from CTTH in children; nevertheless, this genetic factor may be involved in the phenotypic expression, as pressure hypersensitivity was greater in those CTTH children with the Met/Met genotype. (Pediatr Res 70: 395-399, 2011)
\end{abstract}

$\mathrm{T}$ ension-type headache is the most common form of head pain in both adult (1) and child (2) population. Although there has been an increasing knowledge in the pathogenesis of tension-type headache (TTH), the pathoanatomical mechanisms are not completely understood (3). As TTH has several negative repercussions for children, further studies are needed $(4,5)$.

Genetic epidemiological studies have investigated the familial aggregation of TTH. In fact, in individuals with chronic TTH (CTTH), first-degree relatives have a 3.1-fold significantly increased risk of suffered from CTTH $(6,7)$, suggesting a possible role of genetic factors in CTTH. The catechol-O-

Received January 21, 2011; accepted April 7, 2011

Correspondence: César Fernández de las Peñas, Ph.D., Facultad de Ciencias de la Salud, Universidad Rey Juan Carlos, Avenida de Atenas s/n, 28922 Alcorcón, Madrid, Spain; e-mail: cesar.fernandez@urjc.es

Supported by grants from the Universidad Rey Juan Carlos and Comunidad de Madrid (reference CCG10-URJC BIO-5011). methyltransferase (COMT) gene is one of the several potential headache genetic determinants. The COMT is an enzyme involved in the metabolic degradation of dopamine, norepinephrin, and epinephrine (8). Several studies indicate that the genetic polymorphism due to a $\mathrm{G} \rightarrow \mathrm{A}$ substitution at codon 158 of the COMT gene, which leads to a valine (Val) to methionine (Met) substitution, results in differences in COMT activity: a valine (Val) at codon 158 results in a heat-stable, high-activity COMT variant ( Val/Val), whereas a methionine (Met) at this position ( $\mathrm{Val} / \mathrm{Met}$, or Met/Met) results in heatlabile, low-activity variants $(9,10)$. Zubieta et al. (11) demonstrated that individuals with $\mathrm{Val} / \mathrm{Val}$ genotype have a 3- to 4-fold higher activity of the COMT enzyme and reduced pain sensitivity compared with those with the Met/Met genotype, suggesting that the presence of Met/Met genotype may predispose for chronic pain.

Although it might be possibly an influence of COMT polymorphisms for chronic pain, few studies have examined the association between Val158Met polymorphisms and headache. In addition, results from the studies are controversial, as one study found that the frequency of Met/Met genotype was higher in subjects with migraine (12), whereas others reported no differences in genotype distribution and allele frequency of COMT polymorphisms between migraine and healthy subjects $(13,14)$. Another study did not find relationship of Val158Met polymorphism at the COMT in individuals with medication overuse chronic daily headache, episodic migraine without aura, and without analgesic abuse and healthy controls (15). Therefore, more studies are required.

In addition, mechanical hypersensitivity is considered a clear manifestation of hyperexcitability of peripheral and central nociceptive pain pathways in TTH, in both adults (16) and children (17). Jensen et al. (18) have recently reported that the COMT Val158Met genotype has a marked influence on the pain experience following standard heat pain provocations after an opiate injection, suggesting that COMT polymorphisms are involved in pain hypersensitivity.

Abbreviations: ANCOVA, analysis of covariance; COMT, catechol- $O$ methyltransferase; CTTH, chronic tension type headache; PPT, pressure pain threshold; TTH, tension type headache; TTS, total tenderness score 
To the best of the authors' knowledge, no previous study has investigated the possible role of Val158Met polymorphism in the genetic susceptibility to headache and its phenotypic expression in children with CTTH. The aims of this study were to: (1) assess the association between the Val158Met polymorphism in children with CTTH; (2) analyze the relationship between Val158Met polymorphisms and headache clinical pain parameters (intensity, frequency, or duration); and (3) investigate whether Val158Met polymorphisms are related to widespread pressure pain hypersensitivity in children with CTTH. We hypothesized that Met/Met genotype of the Val158Met polymorphism is more prevalent in children with CTTH compared with healthy children and associated with greater widespread pressure pain hypersensitivity.

\section{METHODS}

Participants. Consecutive children diagnosed with TTH by an experienced neuropediatrist from the Neurology Department of Hospital Quirón were screened for eligibility criteria. In all children, headache features, temporal profile of the headache, and family history were assessed. To be included, children had to describe all the characteristics typical of CTTH (ICHD-II) (19): bilateral location, pressing or tightening pain, mild/moderate intensity ( $\leq 6$ on a numerical pain rate scale), and no aggravation of headache during physical activity. No children reported vomiting or nausea during pain attacks. Other primary headaches, medication overuse, and secondary headaches were excluded. None of the participating children were taken prophylactic drugs at the time of the study. Children with diagnosis of depression were also excluded. In addition, age- and sex-matched children without history of head, neck, or shoulder pain symptoms were recruited from volunteer who responded to a local announcement. Again, history of headaches on first- and second-degree relatives was assessed. Ethical approval was granted by the Local Ethics Committee (institution: Universidad Rey Juan Carlos). Written informed consent was obtained from both children and parents, and procedures were conducted according to the Declaration of Helsinki.

Self-reported measures. Children, with the help of their parents completed a headache diary for $4 \mathrm{wk}$ to confirm and complement the diagnosis (20). An 11-point numerical pain rate scale (21) (NPRS; 0: no pain, 10: maximum pain) was used to evaluate headache intensity. The headache diary was used to calculate the following variables: (1) headache intensity, calculated from the mean of the NPRS of the days with headache; (2) mean headache frequency, calculated by dividing the number of days with headache by the number of the analyzed weeks (d/wk), and (3) headache duration, calculated by dividing the sum of the total hours of headache by the number of days with headache $(\mathrm{h} / \mathrm{d})$.

Genetic samples. Nonstimulated whole saliva samples were collected into collection tubes (passive drooling technique) from children according to standardized procedures. Saliva collections were made with children seated, leaning forward and with their heads tilted down. All saliva sampling was performed between 9 and 11 am on days when children were headache-free. Children were asked not to eat or drink or chew gum for $1 \mathrm{~h}$ before the collection sampling. Immediately after collection, samples were centrifuged at $3000 \mathrm{rpm}$ for $15 \mathrm{~min}$ to obtain the cell sediment and they were stored at $-20^{\circ} \mathrm{C}$ until main analysis. We prefer to use saliva instead of blood sampling because salivary collection is a noninvasive, stress-free, and ethic suitable assessment method for children which has been previously used $(22,23)$.

Laboratory technicians were blinded to the children's condition. Genomic DNA was extracted from saliva cell sediments using the "Genomic DNA extraction and purification Kit" (Real Molecular Biology) following the manufacturer's instructions. The single Val158Met (rs4633) nucleotide polymorphism was genotyped using a TaqMan ${ }^{\circledR}$ Drug Metabolism Genotyping Assays on a Real Time PCR ABI Prism 7000 Sequence Detection System (Applied Biosystem, USA) in the Genomic Unit at the Centro de Apoyo Tecnológico (Spain). The three possible halotypes were associated with different fluorescent dyes to proper identification of the different genotype forms: Val/Val (H/H), Met/Val/ (L/H), or Met/Met (L/L).

Pressure pain thresholds (PPT). PPT is defined as the minimal amount of pressure where a sensation of pressure changes to pain (24). An electronic algometer (Somedic AB, Farsta, Sweden) was used to measure PPT (kPa). The pressure was applied at a rate of $30 \mathrm{kPa} / \mathrm{s}$. All participants were instructed to press switch when the sensation changed from pressure to pain. The mean of three trials (intraexaminer reliability) was calculated and used for the main analysis. A 30-s resting period was allowed between each trial. The reliability of pressure algometry has been found to be high (intraclass correlation coefficient: $0.91,95 \%$ CI $0.82-0.97)(25)$.

Children attended a preliminary session for familiarization with the pressure test procedures and they were explored in days headache-free. PPT levels were measured bilaterally over the temporalis muscle, upper trapezius muscle, second metacarpal, and tibialis anterior muscle by an assessor blinded to the children's condition. The order of assessment was randomized on each child.

Total tenderness score (TTS). The TTS was used to assess pericranial tenderness: eight pairs of muscles and tendon insertions (masseter, temporal, frontal, trapezius, sternocleidomastoid, and suboccipital muscles, coronoid, and mastoid processes) were palpated (26). Manual palpation was done with small rotational movements of the assessors' second and third fingers during $4-5 \mathrm{~s}$. Tenderness was scored on a four-point $(0-3)$ scale at each location. The TTS is calculated from the sum of scores from both sides (total maximum score: 48) (26).

Statistical analysis. Data were analyzed with the SPSS statistical package (17.0 Version). Results are expressed as mean and 95\% CI. The KolmogorovSmirnov test showed that quantitative variables showed a normal distribution of the data $(p>0.05)$. Comparisons of genotype distribution and allele frequency between groups were performed on raw frequencies using a $\chi^{2}$ test. A $\chi^{2}$ analysis of the Hardy-Weinberg equilibrium for the genotypes was conducted to determine whether the allele frequencies were stable within patients and controls. A one-way mixed ANOVA was used to compare headache clinical pain parameters and TTS according to the Val158Met polymorphism genotype (Val/Val, Val/Met, and Met/Met) within children with CTTH. A three-way mixed analysis of covariance (ANCOVA) test was used to investigate differences in PPT over each point (temporalis muscle, upper trapezius muscle, second metacarpal, and tibialis anterior muscle) with side (dominant/nondominant) as within-subject factor, group (CTTH, or controls) and Val158Met polymorphism genotype (Val/Val, Val/Met, and Met/Met) as the between-subject factors, and gender as covariate. Post hoc analyses comparisons were done with the Tukey test. The statistical analysis was conducted at a 95\% CI. A $p$ value less than 0.05 was considered statistically significant.

\section{RESULTS}

Clinical data of the sample. Ninety-six $(n=96)$ consecutive children presenting with headache between May 2009 and November 2010 were screened for eligibility criteria. Twentysix $(27 \%)$ were excluded: migraine $(n=12)$, hemicranial headache $(n=5)$, and high levels (BDI-II $>9$ point) of depression and anxiety $(n=9)$. Finally, 70 children, 19 boys and 51 girls, aged 6-11 y old (mean: $9 \pm 2$ y old) with CTTH associated with pericranial tenderness (TTS 19.4, 95\% CI 17.9-20.8) agreed to participate. In this sample, headache history was 2.2 y (95\% CI $1.9-2.4$ y), the mean intensity per episode was 5.0 (95\% CI 4.8-5.3), the mean headache period per day was $3.6 \mathrm{~h}(95 \% \mathrm{CI} 3.0-4.1 \mathrm{~h})$, and the number of $\mathrm{d} / \mathrm{wk}$ with headache was $4.4(95 \%$ CI $3.9-5.7 \mathrm{~d} / \mathrm{wk})$. A significant positive relation $\left(r_{\mathrm{s}}=0.399, p=0.001\right)$ between frequency and duration of the headache was found: the higher the frequency of the headache, the longer the headache duration. The Beck Depression Inventory (BDI-II) score was 3.6 (95\% CI 3.3-3.8).

In addition, 70 age- and sex-matched children without headache, 19 boys and 51 girls, aged $6-11$ y old (mean: $9 \pm$ 1.8 y) were also included $(p=0.916)$. Twenty-two $(n=22$, $31.5 \%$ ) children with CTTH had a first-degree relative with primary headache, whereas $4(6 \%)$ healthy children had firstdegree and $10(16 \%)$ second-degree relatives with a diagnosis of primary headache.

Distribution of Val158Met polymorphism in the COMT gene. The genotype distributions in children with CTTH and healthy children did not deviate from those expected based on 
the Hardy-Weinberg equilibrium. The distribution of COMT Val158Met genotypes was not significantly different $\left(\chi^{2}=\right.$ 2.192, $p=0.335)$, between children with CTTH and children without headache (Table 1). In addition, the distribution of COMT Val158Met genotypes was not also significantly different $\left(\chi^{2}=0.274, p=0.872\right)$ between boys and girls (Table 2$)$.

Clinical pain parameters and Val158Met polymorphism in children with $\boldsymbol{C T T H}$. A one-way ANOVA test revealed significant differences depending on Val158Met polymorphism genotype for the length of headache pain history $(F=7.277$, $p<0.001)$, but not for headache intensity $(F=0.770, p=$ $0.467)$, frequency $(F=0.832, p=0.568)$, or duration $(F=$ $0.571, p=0.487)$ : children with CTTH with the Met/Met genotype showed a longer headache history (mean $\pm \mathrm{SD}$ : $3.8 \pm 0.4 \mathrm{y})$ compared with those children with Met/Val genotype (mean $\pm \mathrm{SD}: 2.0 \pm 1.1 \mathrm{y}, p=0.001)$ and those with $\mathrm{Val} / \mathrm{Val}$ genotype (mean $\pm \mathrm{SD}: 2.1 \pm 1.0 \mathrm{y}, p=0.002$ ).

No significant differences $(F=0.508, p=0.604)$ for TTS between children with CTTH with the L/L (Met/Met) genotype (mean $\pm \mathrm{SD}: 19.8 \pm 7.1)$, children with $\mathrm{L} / \mathrm{H}(\mathrm{Met} / \mathrm{Val})$ genotype (mean $\pm \mathrm{SD}: 18.9 \pm 5.2$ ) and those with $\mathrm{H} / \mathrm{H}$ (Val/Val) genotype (mean $\pm \mathrm{SD}: 21.6 \pm 9.2$ ) were found.

Pressure pain sensitivity and Val158Met polymorphism in the COMT gene. The three-way ANCOVA revealed significant differences between both groups, but not between side, or Val158Met Polymorphism genotype for PPT levels over the temporalis (group: $F=522.894, p<0.001$; side: $F=0.475$, $p=0.491$; Val158Met polymorphism: $F=2.589, p=0.089$ ), upper trapezius (group: $F=423.613, p<0.001$; side: $F=$ $0.680, p=0.410$; Val158Met polymorphism: $F=2.702, p=$ 0.069), second metacarpal (group: $F=491.741, p<0.001$; side: $F=0.237, p=0.627$; Val158Met polymorphism: $F=$ $1.234, p=0.293$ ), and the tibialis anterior muscle (group: $F=$ 542.520, $p<0.001$; side: $F=0.076, p=0.784$; Val158Met

Table 1. Distribution of Val158Met genotypes and alleles of the catechol-O-methyltransferase gene in children with chronic tension type headache and children without headache

\begin{tabular}{ccc}
\hline & $\begin{array}{c}\text { Children with CTTH } \\
(n=70)\end{array}$ & $\begin{array}{c}\text { Healthy children } \\
(n=70)\end{array}$ \\
\hline Genotypes & & \\
H/H (Val/Val) & $22(31 \%)$ & $23(33 \%)$ \\
L/H (Met/Val) & $43(61 \%)$ & $36(52 \%)$ \\
L/L (Met/Met) & $5(8 \%)$ & $11(15 \%)$ \\
Alleles & & \\
Val & $87(62 \%)$ & $82(59 \%)$ \\
Met & $53(38 \%)$ & $58(41 \%)$ \\
\hline
\end{tabular}

polymorphism: $F=1.392, p=0.393)$. Children with CTTH showed bilateral lower PPT levels in all assessed points compared with controls $(p<0.001)$.

In addition, a significant interaction between group and Val158Met polymorphism genotype was found for PPT levels over the temporalis $(F=5.782 ; p=0.003)$ and upper trapezius $(F=6.808 ; p=0.001)$ muscles. Children with CTTH with the L/L (Met/Met) genotype showed lower PPT levels in both points compared with children with CTTH with L/H (Met/Val) genotype and those with the $\mathrm{H} / \mathrm{H}(\mathrm{Val} / \mathrm{Val})$ genotype $(p<0.01)$. No significant differences in PPT depending on Val158Met polymorphism genotype were found within healthy children. No other interaction was found $(p>0.402)$.

Finally, the inclusion of gender as covariate did not influence PPT in the temporalis $(F=1.569, p=0.210)$, upper trapezius $(F=1.400, p=0.238)$, second metacarpal $(F=$ $0.933, p=0.395)$, and tibialis anterior $(F=1.155, p=$ 0.283). Table 3 summarizes PPT according to Val158Met polymorphism genotype and gender in children with CTTH, whereas Table 4 shows PPT levels according to Val158Met polymorphism genotype and gender in healthy children.

\section{DISCUSSION}

This study found no differences in the genotype distribution and allele frequency of the Val158Met polymorphism between children with CTTH and healthy children. In addition, the presence of the Met/Met genotype was associated with a longer headache history and lower PPT levels over the upper trapezius and temporalis muscles in children with CTTH, but not in controls.

Current findings suggest that Val158Met COMT polymorphisms do not appear to be involved in predisposition to suffer from CTTH in children. In accordance with our results, the Val158Met polymorphism was not associated with neuropathic pain (27), chronic musculoskeletal pain (28), chronic widespread pain $(29)$, or migraine $(13,14)$. On the contrary, other studies have associated the Val158Met polymorphism to fibromyalgia (30) or temporomandibular pain (31). It is possible that Val158Met polymorphisms are more related to specific pain syndromes rather than to chronic pain in general. For instance, two other different genetic haplotypes of the COMT gene have been found to be involved in pain perception in temporomandibular pain (31), which may be relevant for headaches. In addition, there is evidence demonstrating that fibromyalgia syndrome and CTTH are usually comorbid in adults $(32,33)$. We do not know if Val158Met polymorphisms can play a role in those headache patients who have

Table 2. Distribution of Val158Met genotypes and alleles of the catechol-O-methyltransferase gene in children with chronic tension type headache and children without headache separated by gender

\begin{tabular}{|c|c|c|c|c|c|c|}
\hline \multirow[b]{2}{*}{ Genotypes } & \multicolumn{3}{|c|}{ Male } & \multicolumn{3}{|c|}{ Female } \\
\hline & $\begin{array}{c}\mathrm{H} / \mathrm{H} \\
(\mathrm{Val} / \mathrm{Val})\end{array}$ & $\begin{array}{c}\mathrm{L} / \mathrm{H} \\
(\mathrm{Met} / \mathrm{Val})\end{array}$ & $\begin{array}{c}\mathrm{L} / \mathrm{L} \\
\text { (Met/Met) }\end{array}$ & $\begin{array}{c}\mathrm{H} / \mathrm{H} \\
(\mathrm{Val} / \mathrm{Val})\end{array}$ & $\begin{array}{c}\mathrm{L} / \mathrm{H} \\
(\mathrm{Met} / \mathrm{Val})\end{array}$ & $\begin{array}{c}\mathrm{L} / \mathrm{L} \\
\text { (Met/Met) }\end{array}$ \\
\hline СТTH & $7(18 \%)$ & $11(30 \%)$ & $1(2 \%)$ & $15(14 \%)$ & $32(32 \%)$ & $4(4 \%)$ \\
\hline Controls & $5(14 \%)$ & $8(21 \%)$ & $6(15 \%)$ & $18(18 \%)$ & $28(27 \%)$ & $5(5 \%)$ \\
\hline Alleles & \multicolumn{2}{|c|}{ Val } & Met & \multicolumn{2}{|c|}{ Val } & Met \\
\hline СТTH & \multicolumn{2}{|c|}{$25(32 \%)$} & $13(17 \%)$ & \multicolumn{2}{|c|}{$62(30 \%)$} & $40(20 \%)$ \\
\hline Controls & \multicolumn{2}{|c|}{$18(24 \%)$} & $20(27 \%)$ & \multicolumn{2}{|c|}{$64(32 \%)$} & $38(18 \%)$ \\
\hline
\end{tabular}


Table 3. Differences in pressure pain thresholds $(\mathrm{kPa})$ over the temporalis, upper trapezius, and tibialis anterior muscles and over the second metacarpal in children with chronic tension type headache depending on Val158Met polymorphism in the catechol-Omethyltransferase gene

\begin{tabular}{|c|c|c|c|c|}
\hline & Temporalis* & Upper trapezius* & Second metacarpal & Tibialis anterior \\
\hline \multicolumn{5}{|c|}{$\begin{array}{l}\text { Children with CTTH with } \mathrm{H} / \mathrm{H} \\
\text { (Val/Val) genotype }(n=22)\end{array}$} \\
\hline Dominant & $177.5 \pm 30.2(164.0-195.1)$ & $155.5 \pm 34.1(138.8-161.6)$ & $260.2 \pm 26.5(243.3-271.3)$ & $311.3 \pm 28.8(294.2-329.9)$ \\
\hline Nondominant & $177.4 \pm 31.1(165.4-192.8)$ & $163.9 \pm 28.1(152.4-177.6)$ & $260.6 \pm 27.3(244.5-273.5)$ & $308.3 \pm 31.7(294.2-327.4)$ \\
\hline \multicolumn{5}{|c|}{$\begin{array}{l}\text { Children with CTTH with } \mathrm{L} / \mathrm{H} \\
\text { (Met/Val) genotype }(n=43)\end{array}$} \\
\hline Dominant & $185.1 \pm 29.8(178.5-199.1)$ & $146.7 \pm 33.1(138.8-159.6)$ & $252.3 \pm 26.7(243.5-264.0)$ & $312.2 \pm 25.1(300.2-326.4)$ \\
\hline Nondominant & $189.8 \pm 31.9(182.9-203.1)$ & $148.7 \pm 27.1(141.4-162.8)$ & $265.9 \pm 22.9(256.6-277.9)$ & $312.3 \pm 26.2(299.6-324.0)$ \\
\hline \multicolumn{5}{|c|}{$\begin{array}{l}\text { Children with CTTH with } \mathrm{L} / \mathrm{L} \\
\text { (Met/Met) genotype }(n=5)\end{array}$} \\
\hline Dominant & $159.0 \pm 26.3(141.3-190.1)$ & $127.4 \pm 24.4(110.2-145.2)$ & $272.2 \pm 16.6(244.1-304.5)$ & $296.0 \pm 28.1(275.7-322.9)$ \\
\hline Nondominant & $156.0 \pm 32.1(140.7-189.0)$ & $129.9 \pm 29.9(112.5-144.8)$ & $265.8 \pm 35.4(238.4-300.9)$ & $292.6 \pm 22.9(273.0-317.9)$ \\
\hline
\end{tabular}

Values \pm standard deviation are expressed as mean (95\% confidence interval).

* Significant lower PPTs in L/L (Met/Met) when compared with H/H (Val/Val) and L/H (Met/Val) genotypes (three-way ANCOVA test).

Table 4. Differences in pressure pain thresholds $(\mathrm{kPa})$ over the temporalis, upper trapezius, and tibialis anterior muscles and over the second metacarpal in children without headache depending on Val158Met polymorphism in the catechol-O-methyltransferase gene

\begin{tabular}{|c|c|c|c|c|}
\hline & Temporalis & Upper trapezius & Second metacarpal & Tibialis anterior \\
\hline \multicolumn{5}{|c|}{$\begin{array}{l}\text { Healthy children with } \mathrm{H} / \mathrm{H} \\
\text { (Val/Val) genotype } \\
(n=23)\end{array}$} \\
\hline Dominant & $300.1 \pm 26.1(284.3-313.4)$ & $255.0 \pm 20.1(239.5-268.8)$ & $426.5 \pm 21.1(412.6-441.7)$ & $507.1 \pm 45.1(482.0-519.0)$ \\
\hline Nondominant & $299.4 \pm 16.7(284.5-312.9)$ & $249.8 \pm 17.5(236.8-262.9)$ & $427.1 \pm 33.4(413.4-443.6)$ & $525.8 \pm 32.7(506.8-541.3)$ \\
\hline \multicolumn{5}{|c|}{$\begin{array}{l}\text { Healthy children with } \mathrm{L} / \mathrm{H} \\
\text { (Met/Val) genotype } \\
(n=36)\end{array}$} \\
\hline Dominant & $304.2 \pm 21.7(291.2-317.7)$ & $254.2 \pm 15.8(241.4-262.9)$ & $425.1 \pm 26.1(415.2-436.6)$ & $520.5 \pm 27.6(507.3-534.6)$ \\
\hline Nondominant & $306.0 \pm 22.3(293.5-318.4)$ & $255.4 \pm 14.9(247.4-266.6)$ & $419.8 \pm 26.8(409.3-431.4)$ & $514.2 \pm 32.3(502.9-528.3)$ \\
\hline \multicolumn{5}{|c|}{$\begin{array}{l}\text { Healthy children with } \mathrm{L} / \mathrm{L} \\
\text { (Met/Met) genotype } \\
(n=11)\end{array}$} \\
\hline Dominant & $322.4 \pm 26.0(299.1-338.9)$ & $254.2 \pm 15.9(236.3-276.3)$ & $435.5 \pm 12.4(413.5-453.1)$ & $507.5 \pm 22.2(487.0-537.6)$ \\
\hline Nondominant & $302.6 \pm 12.4(282.9-321.7)$ & $265.2 \pm 28.2(244.1-279.6)$ & $421.0 \pm 11.4(400.2-441.2)$ & $509.2 \pm 13.7(487.2-534.2)$ \\
\hline
\end{tabular}

comorbid fibromyalgia. Therefore, future studies including different subgroups of individuals with CTTH and different co-morbid conditions would help to clarify these issues.

The fact that Val158Met polymorphisms were not involved in CTTH in children does not negate the role of genetics in this condition. It is known that the interactions of the dopaminergic system are highly complex, as the control of dopamine activity can be determined by several enzymes. For instance, the study by Park et al. (34) have suggested that serotonergic activity may be involved CTTH with the 5-HTT-gene-linked polymorphic gene (5-HTTLPR) being one of the genetically contributing factors. Therefore, future studies investigating the role of other genetic components in children with CTTH are guarantied.

We found that children with CTTH with the Met/Met genotype exhibited longer length of headache history and greater pressure pain hypersensitivity in head and neck muscles. These findings were not replicated in healthy children, which suggest that the Val158Met polymorphism may be involved in the phenotypic expression of CTTH. Our results are similar to those previously reported by Park et al. (13) in individuals with migraine without aura where the presence of the $\mathrm{L}$ allele was associated with a higher headache pain and more common associated nausea and/or vomiting. Similarly, García-Fructuoso et al. (35) found that fibromyalgia patients with the Met/Met genotype showed a more severe form of the disease when compared with those with the Val/Val genotype. Determining the mechanisms involved in the relationship between Val158Met polymorphisms and pressure hypersensitivity in children with CTTH are beyond the scope of this study. In addition, we should recognize the small number of children with CTTH presenting with Met/Met genotype in the current sample. Nevertheless, the percentage of children with CTTH (8\%) and healthy children (15) with the Met/Met genotype was the same than the percentages found in previous studies in adult populations $(13,27,30)$. Future studies are needed to elucidate the role of Val158Met polymorphisms in pressure hypersensitivity in different headaches.

Finally, some points of this study should be considered with caution. First, we included children with CTTH. Therefore, it should be interesting to analyze the role of the Val158Met polymorphism in other primary headaches (e.g. migraine). Second, a greater sample size would be needed to confirm current results, as the number of children with CTTH with Met/Met genotype $(n=5)$ was relatively small considering that our sample size was 70. Third, we only investigated the single Val158Met (rs4633) nucleotide polymorphism. Future studies should include greater number of Val158Met polymorphisms in addition to other genetic factors (e.g. 5-HTTLPR). 
We found no differences in the genotype distribution and allele frequency of the Val158Met polymorphism between children with CTTH and controls. The presence of the Met/ Met genotype was associated with a longer headache history and greater pressure pain hypersensitivity in the neck and head muscles in children with CTTH, but not in controls. Our results suggest that the Val158Met COMT polymorphism does not appear to be involved in predisposition to suffer from CTTH in children, although it may be involved in the phenotypic expression of CTTH. Future studies are needed to elucidate these relationships.

\section{REFERENCES}

1. Andlin-Sobocki P, Jonsson B, Wittchen HU, Olesen J 2005 Cost of disorders of the brain in Europe. Eur J Neurol 12:1-27

2. Unalp A, Dirik E, Kurul S 2007 Prevalence and clinical findings of migraine and tension-type headache in adolescents. Pediatr Int 49:943-949

3. Fernández-de-las-Peñas C, Schoenen J 2009 Chronic tension type headache: what's new? Curr Opin Neurol 22:254-261

4. Hershey A, Kabbouche M, Powers S 2006 Tension-type headache in the young. Curr Pain Headache Rep 10:467-470

5. Anttila P 2006 Tension-type headache in childhood and adolescence. Lancet Neurol 5:268-274

6. Ostergaard S, Russell MB, Bendtsen L, Olesen J 1997 Comparison of first degree relatives and spouses of people with chronic tension headache. BMJ 314:1092-1093

7. Russell MB, Iselius L, Østergaard S, Olesen J 1998 A complex segregation analysis of chronic tension-type headache. Hum Genet 102:138-140

8. Männistö PT, Kaakkola S 1999 Catechol- $O$-methyltransferase (COMT): biochemistry, molecular biology, pharmacology, and clinical efficacy of the new selective COMT inhibitors. Pharmacol Rev 51:593-628

9. Strous RD, Bark N, Woerner M, Lachman HM 1997 Lack of association of a functional catechol- $O$-methyltransferase gene polymorphism in schizophrenia. Biol Psychiatry 41:493-495

10. Syvänen AC, Tilgmann C, Rinne J, Ulmanen I 1997 Genetic polymorphism of catechol- $O$-methyltransferase (COMT): correlation of genotype with individual variation of S-COMT activity and comparison of the allele frequencies in the normal population and Parkinsonian patients in Finland. Pharmacogenetics 7:65-71

11. Zubieta JK, Heitzeg MM, Smith YR, Bueller JA, Xu K, Xu Y, Koeppe RA, Stohler CS, Goldman D 2003 COMT val158 met Genotype affects $\mu$-opioid neurotransmitter responses to a pain stressor. Science 299:1240-1243

12. Emin Erdal M, Herken H, Yilmaz M, Bayazit YA 2001 Significance of the catechol- $O$-methyltransferase gene polymorphism in migraine. Brain Res Mol Brain Res 94:193-196

13. Park JW, Lee KS, Kim JS, Kim YI, Shin HE 2007 Genetic contribution of catechol-O-methyltransferase polymorphism in patients with migraine without aura. J Clin Neurol 3:24-30

14. Hagen K, Pettersen E, Stovner LJ, Skorpen F, Zwart JA 2006 The association between headache and Val158Met polymorphism in the catechol- $O$-methyltransferase gene: the HUNT study. J Headache Pain 7:70-74

15. Montagna P, Cevoli S, Marzocchi N, Pierangeli G, Pini LA, Cortelli P, Mochi M 2003 The genetics of chronic headaches. Neurol Sci 24:S51-S56

16. Fumal A, Schoenen J 2008 Tension-type headache: current research and clinical management. Lancet Neurol 7:70-83
17. Fernández-de-Las-Peñas C, Fernández-Mayoralas DM, Ortega-Santiago R, AmbiteQuesada S, Gil-Crujera A, Fernández-Jaén A 2010 Bilateral wide-spread, mechanical pain sensitivity in children with frequent episodic tension-type headache suggesting impairment in central nociceptive processing. Cephalalgia 30:1049-1055

18. Jensen KB, Lonsdorf TB, Schalling M, Kosek E, Ingvar M 2009 Increased sensitivity to thermal pain following a single opiate dose is influenced by the COMT val158met Polymorphism. PLoS ONE 4:e6016

19. Headache Classification Subcommittee of the International Headache Society 2004 The International Classification of Headache Disorders:2nd edition. Cephalalgia 24:9-160

20. Phillip D, Lyngberg AC, Jensen R 2007 Assessment of headache diagnosis: A comparative population study of a clinical interview with a diagnostic headache diary. Cephalalgia 27:1-8

21. Jensen MP, Turner JA, Romano JM, Fisher L 1999 Comparative reliability and validity of chronic pain intensity measures. Pain 83:157-162

22. Frigerio A, Ceppi E, Rusconi M, Giorda R, Raggi ME, Fearon P 2009 The role played by the interaction between genetic factors and attachment in the stress response in infancy. J Child Psychol Psychiatry 50:1513-1522

23. George SZ, Dover GC, Wallace MR, Sack BK, Herbstman DM, Aydog E, Fillingim RB 2008 Biopsychosocial influence on exercise-induced delayed onset muscle soreness at the shoulder: pain catastrophizing and catechol- $O$-Methyltransferase (COMT) diplotype predict pain ratings. Clin J Pain 24:793-801

24. Vanderweeën L, Oostendorp RA, Vaes P, Duquet W 1996 Pressure algometry in manual therapy. Man Ther 1:258-265

25. Chesterton LS, Sim J, Wright CC, Foster NE 2007 Interrater reliability of algometry in measuring pressure pain thresholds in healthy humans, using multiple raters. Clin J Pain 23:760-766

26. Langemark M, Olesen J 1987 Pericranial tenderness in tension headache. A blind controlled study. Cephalalgia 7:249-255

27. Armero P, Muriel C, Santos J, Sanchez-Montero FJ, Rodriguez RE, GonzalezSarmiento R 2005 COMT (Val158Met) polymorphism is not associated to neuropathic pain in a Spanish population. Eur J Pain 9:229-232

28. Hagen K, Pettersen E, Stovner LJ, Skorpen F, Zwart JA 2006 No association between chronic musculoskeletal complaints and Val158Met polymorphism in the Catechol- $O$-methyltransferase gene: the HUNT study. BMC Musculoskelet Disord 7:40

29. Nicholl BI, Holliday KL, Macfarlane GJ, Thomson W, Davies KA, O’Neill TW, Bartfai G, Boonen S, Casanueva F, Finn JD, Forti G, Giwercman A, Huhtaniemi IT, Kula K, Punab M, Silman AJ, Vanderschueren D, Wu FC, McBeth J; European Male Ageing Study Group 2010 No evidence for a role of the catechol-Omethyltransferase pain sensitivity haplotypes in chronic widespread pain. Ann Rheum Dis 69:2009-2012

30. Gürsoy S, Erdal E, Herken H, Madenci E, Alasehirli B, Erdal N 2003 Significance of the catechol- $O$-methyltransferase gene polymorphism in fibromyalgia syndrome. Rheumatol Int 23:104-107

31. Diatchenko L, Slade GD, Nackley AG, Bhalang K, Sigurdsson A, Belfer I, Oldman D, Xu K, Shabalina SA, Shagin D, Max MB, Marakov SS, Maixner W 2005 Genetic basis for individual variations in pain perception and the development of a chronic pain condition. Hum Mol Genet 14:135-143

32. Kanaan RA, Lepine JP, Wessely SC 2007 The association or otherwise of the functional somatic syndromes. Psychosom Med 69:855-859

33. Schoenen J 2004 Tension-type headache and fibromyalgia: what's common, what's different? Neurol Sci 25:S157-S159

34. Park JW, Kim JS, Lee HK, Kim YI, Lee KS 2004 Serotonin transporter polymorphism and harm avoidance personality in chronic tension-type headache. Headache 44:1005-1009

35. García-Fructuoso FJ, Lao-Villadóniga JI, Beyer K, Santos C 2006 [Relationship between catechol- $O$-methyltransferase genotypes and fibromyalgia's severity.] Reumatol Clin 2:168-172 